\title{
Impact of radiology reports on timely tuberculosis diagnosis
}

\author{
Kristen V Dicks, ${ }^{1}$ David P Holland, ${ }^{2,3}$ Myra G Allen, ${ }^{4}$ Ellen R Fortenberry, ${ }^{4}$ \\ Julie Luffman, ${ }_{1}{ }^{4}$ Elizabeth Zeringue, ${ }^{4}$ Jennifer Wheeler, ${ }_{1}{ }^{4}$ Jason E Stout ${ }^{1,4}$
}

${ }^{1}$ Division of Infectious Diseases and International Health, Department of Medicine, Duke University Medical Center, Durham, North Carolina, USA 2Division of Infectious Diseases, Emory University School of Medicine, Atlanta, Georgia, USA

${ }^{3}$ Communicable Disease Prevention Branch, Fulton County Board of Health, Atlanta Georgia, USA

${ }^{4}$ Division of Public Health, North Carolina Department of Health and Human Services, North Carolina Tuberculosis Program, Communicable Disease Branch Raleigh, North Carolina, USA

\section{Correspondence to}

Jason E Stout, Division of Public Health, North Carolina Department of Health and Human Services, North

Carolina Tuberculosis Program, Communicable Disease Branch, Raleigh 27710, North Carolina, USA; jason.stout@dm.duke.edu

This work was previously presented as a poster at the 2018 National Tuberculosis Controllers Association Meeting (Palm Springs, California).

Received 3 July 2018 Revised 16 August 2018 Accepted 26 August 2018 Published Online First 19 September 2018

\section{ABSTRACT}

Purpose As tuberculosis becomes less common in higher income countries, clinician familiarity with the disease is declining. Little is known about how chest radiograph interpretations affect tuberculosis care. We sought to determine how tuberculosis-related terminology in an initial chest radiograph reading impacted patient care.

Study design We examined a retrospective cohort of patients with pulmonary tuberculosis in North Carolina from 1 January 2011 to 31 December 2014. Tuberculosis-related terminology was categorised into four mutually exclusive categories. The primary outcomes of interest were the time from the chest radiograph to (1) obtaining the first sputum specimen for acid-fast smear and mycobacterial culture, and (2) initiation of antituberculous treatment.

Results Of 550 available chest radiograph reports, 175 (31.8\%) contained the word 'tuberculosis', 30 (5.5\%) contained the word 'mycobacteria' or 'granulomatous', $43(7.8 \%)$ contained the word 'cavity', and 301 (54.7\%) had none of the above terms mentioned. Patients with the word 'tuberculosis' in the radiology report had a significantly shorter time to collection of the initial sputum specimen for acid-fast smear and mycobacterial culture (median 2 days) and to the start of antituberculous treatment (median 4 days) than patients with none of the keywords. Use of the term 'cavity' in the report was associated with a shorter time to initiation of antituberculous treatment (median 4 days) than if none of the keywords were used.

Conclusion Chest radiograph reports that contained keywords for pulmonary tuberculosis, such as 'tuberculosis' or 'cavity', were associated with less time to collection of sputum and antituberculous treatment.

\section{INTRODUCTION}

Chest radiograph is an important diagnostic tool in the evaluation of pulmonary tuberculosis. It has high sensitivity but limited specificity for detecting pulmonary tuberculosis, and is well suited for tuberculosis screening. Pulmonary tuberculosis can present with a spectrum of radiographic findings, from the classic presentation of upper lobe infiltrates or cavitary lesions to the more atypical hilar lymphadenopathy or pleural effusions in a patient with HIV. $^{1-5}$ Because of this range of findings, interobserver variability is high among even experienced radiologists and chest physicians. ${ }^{6-9}$ Regardless, an abnormal chest radiograph in the evaluation of pulmonary tuberculosis is an indication for full diagnostic evaluation and often empiric antituberculous treatment.

Little is known about how chest radiograph interpretations affect the clinical management of tuberculosis. The purpose of this study was to determine how tuberculosis-related terminology in an initial chest radiograph impacted time to obtaining sputum specimens for acid-fast smear and mycobacterial culture testing, and initiation of antituberculous treatment. We hypothesised that in our low-incidence setting, the use of tuberculosis-related terms in the radiology report would be associated with shorter time to obtain diagnostic studies for tuberculosis and to start antituberculous treatment.

\section{MATERIALS AND METHODS}

We examined a retrospective cohort of patients with confirmed pulmonary tuberculosis in North Carolina who were reported to the North Carolina Tuberculosis Control Program from 1 January 2011 to 31 December 2014. Clinical and demographic data, including the date of the initial chest radiograph, were extracted from the North Carolina Electronic Disease Surveillance System. One of the North Carolina tuberculosis nurse consultants (MGA, ERF, JL or EZ) then reviewed the radiology report associated with this initial chest radiograph and extracted the type of facility (categorised as either a hospital, health department or other outpatient facility; other outpatient facility could include a clinic), the name of the radiologist who read the film and tuberculosis-related terminology used in the report. Tuberculosis-related terminology was categorised into four mutually exclusive categories:

1. The word 'tuberculosis' was used in the radiology report in an affirmative manner (eg, 'Suggestive of tuberculosis', 'Suspicious for tuberculosis', 'Tuberculosis should be considered').

2. The word 'tuberculosis' was not used in the radiology report, but either of the words ' $m y$ cobacteria' or 'granulomatous' was used in an affirmative manner.

3. The word 'tuberculosis', 'mycobacteria' or 'granulomatous' was not used in the radiology report, but the word 'cavity' or a variant (eg, 'cavitary', 'cavitation') was used in an affirmative manner.

4. None of the above terms was used in the radiology report.

The primary outcomes of interest were the time (measured in days) from the chest radiograph to (1) obtaining the first sputum specimen for acid-fast smear and mycobacterial culture, and (2) initiation 
Table 1 Demographic and clinical characteristics of patients with pulmonary tuberculosis in North Carolina with chest radiographs obtained prior to initiation of antituberculous treatment and for whom radiology reports were available

\begin{tabular}{lc}
\hline Characteristics & $\mathbf{n}(\%)$ \\
\hline Age in years, mean (SD) & $44.7(21.9)$ \\
\hline Female sex & $193(35.1)$ \\
\hline Born outside the USA & $232(42.2)$ \\
\hline Race & \\
\hline \multicolumn{1}{|c|}{ Asian } & $97(17.6)$ \\
\hline Black & $209(38.0)$ \\
\hline Native American & $22(4.0)$ \\
\hline White & $197(35.8)$ \\
\hline Multiple races & $25(4.5)$ \\
\hline Hispanic ethnicity (can be any race) & $103(18.7)$ \\
\hline Sputum acid-fast smear-positive & $289(52.5)$ \\
\hline Current smoker & $116(21.1)$ \\
\hline HIV seropositive & $22(4.0)$ \\
\hline Diabetes mellitus & $78(14.2)$ \\
\hline
\end{tabular}

of antituberculous treatment. The secondary outcome was the association between the type of facility at which the chest radiograph was obtained and the category of tuberculosis-associated terminology used to describe the report.

Statistical analysis was performed using R V.3.3.1 (R Core Team, Vienna, Austria, 2016). The $\chi^{2}$ test was used to assess the associations between categorical variables, and the Wilcoxon rank-sum test was used to assess the associations between categorical variables and continuous variables. Adjustment of $\mathrm{p}$ values for multiple comparisons was not performed.

\section{RESULTS}

From 1 January 2011 to 31 December 2014, 866 confirmed tuberculosis cases were reported in North Carolina. Of these 866, 682 (78.8\%) had pulmonary disease, with or without extrapulmonary involvement. Of the 682 patients with pulmonary disease, $550(80.6 \%)$ had chest radiographs performed prior to initiation of antituberculous treatment and with available radiology reports in the local health department medical records. The demographic and clinical characteristics of these patients are summarised in table 1. Patients with tuberculosis tended to be male (64.9\%) and of non-white race; slightly over half (52.5\%) had at least one positive acid-fast smear of sputum, and only $4 \%$ were coinfected with HIV. Patients resided in 74 different counties within North Carolina, although close to half (257, 46.7\%) resided in one of six counties that had over 20 cases each during the study period. Initial chest radiographs were most commonly obtained in a hospital setting $(378,68.7 \%)$, followed by outpatient clinics $(126,22.9 \%)$, health departments $(44,8.0 \%)$ and unknown sites $(2,0.4 \%)$. Chest radiographs were interpreted by 428 radiologists; 75 radiologists read two or more films of patients with tuberculosis and only 17 read three or more films of patients with tuberculosis during the study period.

Of the 550 films, 175 (31.8\%) had the word 'tuberculosis' in the radiology report. Of the remainder, $30(5.5 \%)$ had either of the words 'mycobacteria' or 'granulomatous' in the report, 43 $(7.8 \%)$ reports included none of those words but had the word 'cavity' mentioned in the report, and 301 (54.7\%) had none of the above terms mentioned (data for one report were missing). In some cases in which none of the above terms were mentioned, the report would likely have led an informed clinician to believe that tuberculosis was a likely diagnosis, while in others the report was so vague and non-specific that it likely would not have helped a clinician to diagnose tuberculosis. Some examples of radiology reports in which none of the above terms were mentioned are provided in table 2 . The terminology used in the radiology report was associated with the probability that sputum would be acid-fast smear-positive; $61.1 \%$ of patients with the word 'tuberculosis' in the report had a positive smear, $40.0 \%$ of patients with 'mycobacteria' or 'granulomatous' had a positive smear, $74.4 \%$ of patients with 'cavity' had a positive smear, and $45.8 \%$ of patients with none of the terms used in the report had a positive smear ( $p$ value for overall comparison among groups $<0.001$ ). Patients whose chest radiographs were performed in the hospital were less likely to have the word 'tuberculosis' in the report (28.3\%) than patients whose radiographs were performed in the health department $(39.5 \%)$ or outpatient $(40.5 \%)$ settings $(p=0.02$ for comparison among the three settings). Patients with the word 'tuberculosis' in the radiology report had a significantly shorter time to collection of the initial sputum specimen for acidfast smear and mycobacterial culture (median 2 days) and to the start of antituberculous treatment (median 4 days) than patients with none of the words 'tuberculosis', 'mycobacteria', 'granulomatous' or 'cavity' in the report (tables 3 and 4). The use of the term 'cavity' in the report was associated with a shorter time to initiation of antituberculous treatment (median 4 days) than if none of the above terms were used.

\section{DISCUSSION}

This study suggests that the use of tuberculosis-specific terms in chest radiograph reports was associated with more rapid diagnosis and treatment of patients with culture-confirmed

\section{Table 2 Examples of radiology reports in which none of the tuberculosis-related terms were used}

\begin{tabular}{ll}
\hline Example number & Report text \\
\hline 1 & $\begin{array}{l}\text { 'There is a moderate-sized left pleural effusion with associated left lower lung opacities and indistinct opacification left upper lobe....Impression: 1. Moderate } \\
\text { left effusion 2. Left lower lobe opacification 3. Nonspecific left upper lobe opacification'. }\end{array}$ \\
\hline 2 & $\begin{array}{l}\text { 'Interval development of extensive airspace consolidation throughout the left upper lobe, with confluent consolidation in the medial apical portion of the left } \\
\text { upper lobe...Impression: Pneumonia throughout the entire left upper lobe, with confluent consolidation medially in the left apex. Follow-up chest } x \text {-rays to } \\
\text { resolution are suggested'. } \\
\text { 'Stable right IJ [internal jugular] approach dual lumen dialysis type catheter. Interval decreased pulmonary edema. Continued vascular congestion/diffuse } \\
\text { interstitial opacity. Overall ventilation remains decreased...Impression: Interval decreased but not resolved pulmonary edema. Continued low lung volumes'. }\end{array}$ \\
\hline 4 & $\begin{array}{l}\text { 'Worsening pleural effusion. Developing patchy opacity in the right lower lobe but also multifocal particularly in the upper lobe. Cardiomegaly. No } \\
\text { pneumothorax. Normal vascular structures. Volume loss in the right lower lobe. Impression: Predominantly right lower lobe airspace disease with volume loss } \\
\text { but there is also multifocal airspace opacities. Is a right pleural effusion as well'. }\end{array}$ \\
\hline 5 & $\begin{array}{l}\text { 'The cardiomediastinal silhouette is normal. Left upper lobe airspace disease is seen. Left lower lobe and right lung are clear. Impression: Left upper lobe } \\
\text { infiltrate'. }\end{array}$ \\
\hline
\end{tabular}


Table 3 Time to collection of the first sputum specimen for acid-fast bacilli testing according to radiology report findings

\begin{tabular}{lll}
\hline $\begin{array}{l}\text { Findings in radiology } \\
\text { report }\end{array}$ & $\begin{array}{l}\text { Median days to sputum } \\
\text { collection (IQR) }\end{array}$ & P values \\
\hline None & $4(1-15)$ & Reference \\
$\begin{array}{l}\text { 'Mycobacteria' or } \\
\text { 'Granulomatous' }\end{array}$ & $6(3-11)$ & 0.29 \\
'Cavity' & $3(-0.5$ to 6.25) & 0.26 \\
\hline 'Tuberculosis' & $2(1-4)$ & 0.002 \\
& & $\begin{array}{l}(0.002 \text { also compared } \\
\text { with 'Mycobacteria', } 0.69 \\
\text { compared with 'Cavity') }\end{array}$ \\
\hline
\end{tabular}

pulmonary tuberculosis. A patient whose chest radiograph report contained the word 'tuberculosis' had a shorter time to collection of sputum, and a patient whose chest radiograph report contained the word 'tuberculosis' or 'cavity' had a shorter time to the start of antituberculous treatment. Patients who did not have 'tuberculosis', 'mycobacteria', 'granulomatous' or 'cavity' in the chest radiograph report had a delay in antituberculous treatment, which was, on average, three times that of patients with 'tuberculosis' or 'cavity' in the report. Additionally, patients with tuberculosis whose chest radiograph report contained the word 'mycobacteria' or 'granulomatous' did not have a significant difference in time to collection of sputum or the start of antituberculous treatment as compared with patients who did not have these words in the chest radiograph report. These findings demonstrate the importance of using precise language and mentioning important diagnoses in radiology reports. We suspect that in many cases the diagnosis of tuberculosis was initially raised by the radiologist, resulting in more rapid action to evaluate and treat that diagnosis by the treating team.

Many of the radiograph reports represent a missed opportunity for diagnosing pulmonary tuberculosis. Most of the radiographs of patients with tuberculosis did not mention tuberculosis in the report. In fact, half of the radiograph reports did not contain any of the key tuberculosis terms. Some of these reports described findings typical of tuberculosis but did not mention any of the keywords, while others were completely non-specific and unhelpful in diagnosis (table 2). The failure to mention key tuberculosis-related terms in radiology reports likely represents both diagnostic and communication failure on the part of radiologists. Patients whose radiographic report mentioned 'tuberculosis' or 'cavity' were more likely to have a positive sputum acid-fast smear, but not all smear-positive patients had one of these terms included in the report. As a group, patients with smear-positive pulmonary tuberculosis with typical radiographic features are more likely to transmit tuberculosis, and constitute

\begin{tabular}{lll}
\hline $\begin{array}{l}\text { Table } 4 \\
\text { radiology report findings }\end{array}$ & Time to start antituberculous treatment according to \\
\hline $\begin{array}{l}\text { Findings in radiology } \\
\text { report }\end{array}$ & $\begin{array}{l}\text { Median days to start of } \\
\text { antituberculous treatment (IQR) }\end{array}$ & P values \\
\hline None & $12(4-35)$ & Reference \\
$\begin{array}{lcl}\text { 'Mycobacteria' or } & 9(5-37) & 0.91 \\
\text { 'Granulomatous' } & 4(2-6) & <0.001 \\
\text { 'Cavity' } & 4(2-12) & \begin{array}{l}<0.001 \\
\text { 'Tuberculosis' }\end{array} \\
& & 0.48 \text { compared } \\
\text { with 'Cavity') }\end{array}$ \\
\hline
\end{tabular}

the greatest health risk. ${ }^{10}$ Failing to diagnose these patients has huge implications for both the individual and the community.

Both lack of experience and ineffective communication can decrease the diagnostic utility of chest radiographs for pulmonary tuberculosis. ${ }^{6-9}$ The USA is a low-prevalence area for tuberculosis, ${ }^{11}$ which means clinicians and radiologists have decreasing experience with tuberculosis. ${ }^{12}$ There is a need for improved and ongoing tuberculosis education for radiologists to avoid missing cases of tuberculosis. This is especially important for radiologists who serve immunosuppressed populations in whom tuberculosis is more likely to present with atypical findings. ${ }^{1-5}$ Additionally, standardising radiographic interpretations for patients undergoing screening for pulmonary tuberculosis could lead to clearer communication and fewer missed cases. ${ }^{13-17}$

This study has limitations. The retrospective study was limited to what was available in the surveillance database, which was not designed to capture clinical events. Some radiographs were not available for review. It is possible that patients with more severe disease would be started on treatment sooner irrespective of keywords in the radiograph report. There was a low prevalence of HIV in the study cohort; thus, these findings cannot be directly applied to persons with HIV and tuberculosis. However, given that tuberculosis in persons with HIV tends to present in an atypical manner, it is expected that diagnostic and treatment delays would be similar or worse. Further study on the implications of radiograph report keywords for atypical presentations of tuberculosis in immunosuppressed populations is warranted.

In conclusion, chest radiograph reports that contained keywords for pulmonary tuberculosis, such as 'tuberculosis' or 'cavity', were associated with less time to collection of sputum and antituberculous treatment. Further education about tuberculosis and efforts to standardise radiographic interpretations could increase the diagnostic accuracy of radiographs and improve clinical care of patients with pulmonary tuberculosis.

\section{Main messages}

Most patients with culture-confirmed pulmonary tuberculosis did not have any words suggesting the diagnosis in the initial radiology report.

- Lack of tuberculosis-specific terminology in the initial radiology report was associated with delayed diagnosis and treatment.

\section{Current research questions}

Tuberculosis case rates and clinician experience are declining in much of the developed world.

Delayed tuberculosis diagnosis and treatment are common.

Contributors KVD participated in the analysis, drafted the manuscript and revised the manuscript for critical content. DPH participated in the design of the study, participated in the analysis and revised the manuscript for critical content. MGA, ERF $\mathrm{J}$ and EZ participated in the design of the study, collected the data and revised the manuscript for critical content. JW revised the manuscript for critical content. JES participated in the design of the study, participated in the analysis and revised the manuscript for critical content.

Funding The authors have not declared a specific grant for this research from any funding agency in the public, commercial or not-for-profit sectors.

Competing interests None declared.

Patient consent Not required.

Ethics approval North Carolina Department of Health and Human Services Institutional Review Board. 
Provenance and peer review Not commissioned; externally peer reviewed.

Data sharing statement Deidentified data on radiology reports are available from the authors on written request.

\section{REFERENCES}

1 Kisembo HN, Boon SD, Davis JL, et al. Chest radiographic findings of pulmonary tuberculosis in severely immunocompromised patients with the human immunodeficiency virus. Br J Radiol 2012;85:e130-e139.

2 Barnes PF, Bloch AB, Davidson PT, et al. Tuberculosis in patients with human immunodeficiency virus infection. N Engl J Med 1991;324:1644-50.

3 Kosack CS, Spijker S, Halton J, et al. Evaluation of a chest radiograph reading and recording system for tuberculosis in a HIV-positive cohort. Clin Radiol 2017;72:519.e1-519.e9.

4 Geng E, Kreiswirth B, Burzynski J, et al. Clinical and radiographic correlates of primary and reactivation tuberculosis: a molecular epidemiology study. JAMA 2005;293:2740-5.

5 Haramati LB, Jenny-Avital ER, Alterman DD. Effect of HIV status on chest radiographic and CT findings in patients with tuberculosis. Clin Radiol 1997;52:31-5.

6 Kwong JS, Carignan S, Kang EY, et al. Miliary tuberculosis. Diagnostic accuracy of chest radiography. Chest 1996;110:339-42.

7 Zellweger JP, Heinzer R, Touray M, et al. Intra-observer and overall agreement in the radiological assessment of tuberculosis. Int J Tuberc Lung Dis 2006;10:1123-6.

8 Graham S, Das GK, Hidvegi RJ, et al. Chest radiograph abnormalities associated with tuberculosis: reproducibility and yield of active cases. Int J Tuberc Lung Dis 2002;6:137-42.
9 Stout JE, Kosinski AS, Hamilton CD, et al. Effect of improving the quality of radiographic interpretation on the ability to predict pulmonary tuberculosis relapse. Acad Radiol 2010;17:157-62.

10 Lau A, Barrie J, Winter C, et al. Chest radiographic patterns and the transmission of tuberculosis: implications for automated systems. PLoS One 2016;11:e0154032.

11 Hundie GB, Woldemeskel D, Gessesse A. Evaluation of direct colorimetric mtt assay for rapid detection of rifampicin and isoniazid resistance in Mycobacterium tuberculosis. PLoS One 2016;11:e0169188.

12 Miller AC, Polgreen LA, Cavanaugh JE, et al. Missed Opportunities to diagnose tuberculosis are common among hospitalized patients and patients Seen in emergency departments. Open Forum Infect Dis 2015;2:ofv171.

13 Concepcion NDP, Laya BF, Andronikou S, et al. Standardized radiographic interpretation of thoracic tuberculosis in children. Pediatr Radiol 2017;47:1237-48.

14 Marais BJ, Gie RP, Schaaf HS, et al. A proposed radiological classification of childhood intra-thoracic tuberculosis. Pediatr Radiol 2004;34:886-94.

15 Dawson R, Masuka P, Edwards DJ, et al. Chest radiograph reading and recording system: evaluation for tuberculosis screening in patients with advanced HIV. Int J Tuberc Lung Dis 2010;14:52-8.

16 Den Boon S, Bateman ED, Enarson DA, et al. Development and evaluation of a new chest radiograph reading and recording system for epidemiological surveys of tuberculosis and lung disease. Int J Tuberc Lung Dis 2005;9:1088-96.

17 Pinto LM, Pai M, Dheda K, et al. Scoring systems using chest radiographic features for the diagnosis of pulmonary tuberculosis in adults: a systematic review. Eur Respir J 2013;42:480-94. 